\title{
$6.4-19$
}

\section{ON THE DESIGN OF A SOUND SYSTEM FOR A MOBILE AUDIO UNIT}

\author{
F. Lindström ${ }^{a}$, J.-E. Eriksson ${ }^{a}$, M. Dahl ${ }^{b}$, I. Claesson $^{b}$ \\ ${ }^{a}$ Konftel AB, Research and Development, Box 268, S-90106, Umeå, Sweden \\ ${ }^{b}$ Blekinge Institute of Technology, Department of Signal Processing, S-37225, Ronneby, Sweden
}

\begin{abstract}
A mobile audio unit is a wireless, battery-driven unit, the main purpose of which is to reproduce acoustic signals. This kind of unit can be used in conjunction with a home server. For example, a radio station broadcasting can be received from the Internet and fed to the mobile audio unit via a central home server. The market for home servers is expected to grow leading to a possible expansion of the market for this type of mobile audio unit. This paper presents some design aspects for the sound system of an audio unit, adapted to the new demands of the market.
\end{abstract}

\section{INTRODUCTION}

A home server is a central unit for storage of digital media as well as a hub for interconnection between, for example the Internet, TV, PCs, stereos, etc [1]. One type of service provided by a home server is multimedia streaming [2], e.g. the streaming of an audio source obtained from the Internet and channelled to a mobile audio unit. A mobile audio unit used in conjunction with a home server requires a sound system, i.e. an amplifier-loudspeaker system. This paper discusses some design and implementation issues related to such a sound system.

A mobile audio unit for use with a home server, in this paper denoted "the audio unit", should not be to large, have a long battery life-time, low production cost and be able to produce high quality audio. The definition of "high quality" audio is subjective. For the sake of perspective, a summary of characteristics of different types of radio receivers is given in Table 1. Since the audio unit is normally used for stereo sound reproduction, only stereo solutions are considered in this paper.

\section{SYSTEM ARCHITECTURE}

This section presents two typical stereo audio system architecture solutions, see Fig. 1. The solutions are denoted ST-2 and ST-3. Solution ST-2 is a regular 2-channel stereo solution using two loudspeakers. Solution ST-3 is a 3-channel

\begin{tabular}{|c|c|c|c|c|}
\hline Type & Speakers & $\begin{array}{c}\text { Total } \\
\text { Output Power }\end{array}$ & $\begin{array}{c}\text { Frequency } \\
\text { Response }\end{array}$ & $\begin{array}{c}\text { Power } \\
\text { Supply }\end{array}$ \\
\hline \hline Net & Stereo & $>20 \mathrm{~W}$ & $50-18,000 \mathrm{~Hz}$ & Net \\
World & Mono & $400-500 \mathrm{~mW}$ & $300-15,000 \mathrm{~Hz}$ & Battery \\
Normal & Mono & $1 \mathrm{~W}$ & $150-15,000 \mathrm{~Hz}$ & Battery \\
\hline
\end{tabular}

Table 1. Radio receiver characteristics, Net - Net driven radio receivers, World - World radio receivers, Normal $\mathrm{AM} / \mathrm{FM}$ band-radio receivers.

stereo solution using three loudspeakers. The ST-3 solution splits the fullband signals into subbands, amplifies these, and transmits them on one woofer and two tweeters. The ST-2 solution requires a 2-channel amplifier, while solution ST-3 requires a 3-channel amplifier. The amplifier cost for the ST-3 solution is thus higher. If the incoming signal to the ST-3 solution sound unit is a 2-channel stereo signal, there is a need for a cross-over unit for the frequency split [3], which will further increase cost. However, if good reproduction of low frequency sound is desirable, the ST-2 solution will require two large loudspeakers, while the ST-3 solution can use one large woofer and two small tweeters. As a result, the ST-3 solution requires a smaller physical size of the audio unit.

\section{AMPLIFIERS}

In this section the distortion, power consumption and cost of amplifiers are considered. Two types of amplifiers are considered: Class-AB and Class-D. Class-AB amplifiers are analog amplifiers. Class-D amplifiers are digital amplifiers

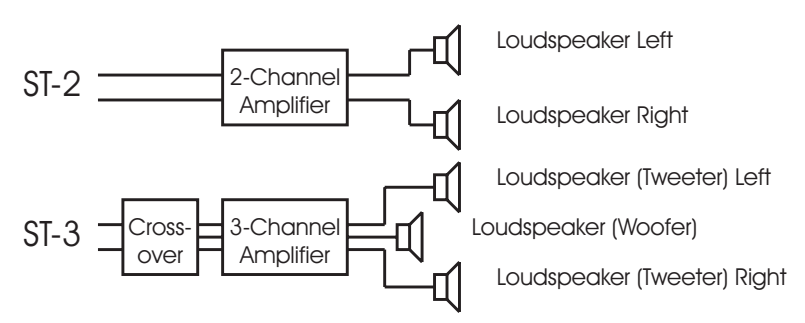

Fig. 1. Two typical architectures, ST-2 and ST-3 


\begin{tabular}{|c|c|c|}
\hline Parameter & Class-AB & Class-D \\
\hline \hline $\begin{array}{c}\text { Distortion (THD+N } \\
\text { at } 1 \mathrm{kHz})\end{array}$ & $0.5 \%$ & $0.5 \%$ \\
\hline $\begin{array}{c}\text { Power (Current consumption } \\
\text { when using a speech signal) }\end{array}$ & $300 \mathrm{~mA}$ & $100 \mathrm{~mA}$ \\
\hline
\end{tabular}

Table 2. Comparison of 2-channel Class-AB and Class$\mathrm{D}$ amplifiers. The values are average values of several 2channel amplifiers from different manufacturers.

which use switching techniques, [4]-[5].

The distortion introduced by the amplifiers is about the same for the Class-AB as the Class-D amplifiers, see Table 2. Further, the distortion of the loudspeakers in the audio unit will probably make the distortion of the amplifiers insignificant.

When comparing current consumption of different amplifiers it is of outmost importance that performance is evaluated when the speakers are driven using proper test-signals. For the type of application considered in this paper, music or a speech signal should be used. Devices that have similar current consumption when using specific test signals, (e.g. tones), may be very different in terms of current consumption when using a speech signal. For Class-AB and Class-D amplifiers, there is a significant difference between the power consumed at normal listening level when driven by a speech signal, see Table 2 .

The price of amplifiers differs depending on such factors as availability and the number of components ordered. During the last few years, the price difference between Class$\mathrm{AB}$ and Class-D amplifiers has decreased. It is possible to find Class-D amplifiers that are less expensive than comparable Class-AB amplifiers, though Class-D amplifiers are generally slightly more expensive.

The difference in power consumption will have a significant influence on the audio unit's usage time. A Class-D amplifier is thus recommended.

\section{LOUDSPEAKER SIZES}

Due to the type of product considered in this paper, the physical size of the loudspeakers is limited. For solution ST2, two different sizes of speakers are appropriate: 2"(inches) and 2.5". The appropriate size of the loudspeakers in the ST3 solution is determined by the crossover, i.e. the bandwidth of the sub-frequencies. A 2.5" woofer and 1" tweeters may, for example, be chosen.

\section{RECOMMENDED SPECIFICATIONS}

In order to produce an audio unit of sufficiently high quality (see Table 1), the following specifications are recommended for the audio unit:

- Frequency response: $80 / 100 \mathrm{~Hz}-15,000 \mathrm{~Hz}$

- Sound pressure: $84 \mathrm{~dB} 1 \mathrm{~W} / 1 \mathrm{~m}$

- Loudspeaker power capacity: $5 \mathrm{~W}$ rms

- Total output power: $4 \mathrm{~W}$ rms

\section{ADDITIONAL RECOMMENDATIONS}

An active highpass filter with a cut-off frequency between $80 \mathrm{~Hz}$ and $100 \mathrm{~Hz}$ may be implemented. This will not reduce the perceived sound quality but will result in lower power consumption.

Automatic loudness control may be implemented. This increase low frequency sound at low volumes. The human hearing system is not as sensitive to low frequency sound at low listening levels as it is at high ones [6]. Automatic loudness control is implemented to counteract this.

\section{CONCLUSIONS}

In this paper, some design aspects of a sound system for a mobile audio unit, for use in conjunction with a home server, have been discussed. Two different system architectures have been proposed. Class-AB and Class-D amplifiers have been compared. Class-D amplifiers are the more attractive thanks to their lower power consumption. Some specifications for the audio unit have been proposed. Finally, some additional recommendations have been made.

\section{REFERENCES}

[1] P. Wallich, "Digital hubbub", IEEE Spectrum, No. 7, pp. 26-31, July 2002.

[2] K. Lee, et. al., "Requirements and referential software architecture of home server based inter-home multimedia collaboration services" IEEE Transactions on Consumer Electronics, vol. 50, no. 1, pp. 145-150, Feb. 2002.

[3] E. Rapoport, F. A. P. Barauqui, A. Petraglia, "Tunable analog loudspeaker crossover network", Proc. of IEEE ISCAS'03, pp. 447-480, 2003.

[4] S. Burrow, D. Grant "Efficiency of low power audio amplifiers and loudspeakers", Transactions on Consumer Electronics, vol. 47, No. 3, pp. 622-630, Aug. 2001.

[5] D. Dapkus, "Class-D audio power amplifiers: an overview”, Proc. of IEEE ICCE'00, pp. 400-401, 2000.

[6] F. A. Everest, Master Handbook of Acoustics, 4th edition, McGraw-Hill, 2001. 